PROCEEDINGS OF THE

AMERICAN MATHEMATICAL SOCIETY

Volume 138, Number 3, March 2010, Pages 809-813

S 0002-9939(09)10083-7

Article electronically published on September 4, 2009

\title{
FURTHER IMPROVEMENTS OF LOWER BOUNDS FOR THE LEAST COMMON MULTIPLES OF ARITHMETIC PROGRESSIONS
}

\author{
SHAOFANG HONG AND SCOTT DUKE KOMINERS
}

(Communicated by Wen-Ching Winnie Li)

\begin{abstract}
For relatively prime positive integers $u_{0}$ and $r$, we consider the arithmetic progression $\left\{u_{k}:=u_{0}+k r\right\}_{k=0}^{n}$.

Define $L_{n}:=\operatorname{lcm}\left\{u_{0}, u_{1}, \ldots, u_{n}\right\}$ and let $a \geq 2$ be any integer. In this paper, we show that for integers $\alpha, r \geq a$ and $n \geq 2 \alpha r$, we have

$$
L_{n} \geq u_{0} r^{\alpha+a-2}(r+1)^{n} .
$$

In particular, letting $a=2$ yields an improvement to the best previous lower bound on $L_{n}$ (obtained by Hong and Yang) for all but three choices of $\alpha, r \geq 2$.
\end{abstract}

\section{INTRODUCTION}

The search for effective estimates on the least common multiples of finite arithmetic progressions began with the work of Hanson [Han72] and Nair [Nai82, who found, respectively, the upper and lower bounds for $\operatorname{lcm}\{1, \ldots, n\}$.

Inspired by this work, Bateman, Kalb, and Stenger [BKS02] and Farhi [Far05], respectively, sought asymptotics and nontrivial lower bounds for the least common multiples of general arithmetic progressions. Farhi Far05 obtained several nontrivial bounds and posed a conjecture which was later confirmed by Hong and Feng HF06]. Additionally, Hong and Feng HF06 obtained an improved lower bound for sufficiently large arithmetic progressions; this result was recently sharpened further by Hong and Yang HY08a. Hong and Yang HY08b and Farhi and Kane [FK09] also obtained some related results regarding the least common multiple of a finite number of consecutive integers. The theorem of Farhi and Kane FK09 was very recently extended to general arithmetic progressions by Hong and Qian HQ09.

In this article, we study finite arithmetic progressions $\left\{u_{k}:=u_{0}+k r\right\}_{k=0}^{n}$ with $u_{0}, r \geq 1$ integers satisfying $\left(u_{0}, r\right)=1$. Throughout, we define

$$
L_{n}:=\operatorname{lcm}\left\{u_{0}, u_{1}, \ldots, u_{n}\right\}
$$

Received by the editors June 12, 2009.

2000 Mathematics Subject Classification. Primary 11A05.

Key words and phrases. Least common multiple, arithmetic progression.

The first author was partly supported by the National Science Foundation of China and by the Program for New Century Excellent Talents in University, Grant No. NCET-06-0785. The second author was partly supported by a U.S. National Science Foundation Graduate Research Fellowship and is the corresponding author. 
to be the least common multiple of the sequence $\left\{u_{k}\right\}_{k=0}^{n}$. The following lower bound on $L_{n}$ was found by Hong and Yang [HY08a].

Theorem 1.1 ([HY08a $)$. Let $\alpha \geq 1$ be an integer. If $n>r^{\alpha}$, then we have $L_{n} \geq u_{0} r^{\alpha}(r+1)^{n}$.

If $r=1$, then the content of Theorem 1.1] is the conjecture of Farhi [Far05] proven by Hong and Feng [HF06. If $\alpha=1$, then Theorem 1.1 becomes the improved lower bound of Hong and Feng [HF06.

In this paper, we sharpen the lower bound in Theorem 1.1 whenever $\alpha, r \geq 2$. In particular, we prove the following theorem which replaces the exponential condition $n>r^{\alpha}$ of Theorem 1.1 with a linear condition, $n \geq 2 \alpha r$.

Theorem 1.2. Let $a \geq 2$ be any given integer. Then for any integers $\alpha, r \geq a$ and $n \geq 2 \alpha r$, we have $L_{n} \geq u_{0} r^{\alpha+a-2}(r+1)^{n}$.

Letting $a=2$, we see that Theorem 1.2 improves upon Theorem 1.1 for all but three choices of $\alpha, r \geq 2$.

The remainder of this paper is organized as follows. In Section 2, we introduce relevant notation and previous results. In Section 3 we prove Theorem 1.2 and, as a corollary, we obtain arbitrarily strong sharpenings of Theorem 1.1 which apply in all but finitely many cases. Then, in Section 4 , we discuss when the condition $n>r^{\alpha}$ is necessary in Theorem 1.1

\section{Notation And PRevious Results}

For any real numbers $x$ and $y$, we say that $y$ divides $x$ if there exists an integer $z$ such that $x=y \cdot z$. If $x$ divides $y$, then we write $y \mid x$. As usual, we let $\lfloor x\rfloor$ denote the largest integer no more than $x$.

Following Hong and Yang [HY08a, we denote, for each integer $0 \leq k \leq n$,

$$
C_{n, k}:=\frac{u_{k} \cdots u_{n}}{(n-k) !}, \quad L_{n, k}:=\operatorname{lcm}\left\{u_{k}, \ldots, u_{n}\right\} .
$$

From the latter definition, we have that $L_{n}=L_{n, 0}$.

The following lemma first appeared in Far05 and was reproven in several sources:

Lemma 2.1 ([Far05, Far07, [HF06]). For any integer $n \geq 1, C_{n, 0} \mid L_{n}$.

From Lemma 2.1, we see immediately that

$$
L_{n, k}=A_{n, k} \frac{u_{k} \cdots u_{n}}{(n-k) !}=A_{n, k} \cdot C_{n, k}
$$

for an integer $A_{n, k} \geq 1$.

Following Hong and Feng [HF06] and Hong and Yang HY08a, we define, for any $n \geq 1$,

$$
k_{n}:=\max \left\{0,\left\lfloor\frac{n-u_{0}}{r+1}\right\rfloor+1\right\} .
$$

Hong and Feng [HF06] proved the following result.

Lemma 2.2 ([HF06]). For all $n \geq 1$ and $0 \leq k \leq n$,

$$
L_{n} \geq L_{n, k_{n}} \geq C_{n, k_{n}} \geq u_{0}(r+1)^{n} .
$$




\section{Proof of the MAin theorem AND COROLlary}

We begin with a lemma which is similar to a key step of the proof of Theorem 1.1 . The proof of this result closely follows the approach of Hong and Yang [HY08a, but simplifies the analysis.

Lemma 3.1. Let $a \geq 2$ be any given integer. Then for any integers $\alpha, r \geq a$ and $n \geq 2 \alpha r$, we have $n-k_{n}>(\alpha+a-2) r$.

Proof. If $n \leq u_{0}$, then by the definition (2) we have $k_{n} \leq 1$. Since $\alpha, r \geq a \geq 2$ and $n \geq 2 \alpha r$, we deduce that $n-k_{n} \geq n-1 \geq 2 \alpha r-1>(\alpha+a-2) r$.

Now, we suppose that $n>u_{0}$. In this case, we have

$$
k_{n}=\left\lfloor\frac{n-u_{0}}{r+1}\right\rfloor+1 ;
$$

it follows that

$$
k_{n} \leq \frac{n-u_{0}}{r+1}+1 \leq \frac{n-1}{r+1}+1=\frac{n+r}{r+1} .
$$

From this, we then see that

$$
n-k_{n} \geq n-\frac{n+r}{r+1}=\frac{(n-1) r}{r+1} \geq \frac{(2 \alpha r-1) r}{r+1} .
$$

However, the assumption $\alpha, r \geq a$ implies that

$$
\begin{aligned}
(2 \alpha r-1)-(r+1)(\alpha+a-2) & =(r-1) \alpha-1-(r+1)(a-2) \\
& \geq a(r-1)-1-(r+1)(a-2) \\
& =2(r-a)+1>0 .
\end{aligned}
$$

Therefore from (4), we infer that

$$
\frac{2 \alpha r-1}{r+1}>\alpha+a-2
$$

The desired result then follows immediately from (3) and (15).

From Lemma 3.1, the proof of Theorem 1.2 follows directly via the same argument as in the endgame of the proof of Theorem 1.1. For completeness, we reproduce this elegant argument here.

Proof of Theorem 1.2. By hypothesis, we have $\alpha, r \geq a \geq 2$ and $n \geq 2 \alpha r$. As a consequence of Lemma 3.1, we therefore obtain that $r^{\alpha+a-2} \mid\left(n-k_{n}\right)$ !. Thus, we may express $\left(n-k_{n}\right)$ ! in the form $r^{\alpha+a-2} \cdot B_{n}=\left(n-k_{n}\right)$ !, with $B_{n} \geq 1$ an integer. If we choose $k=k_{n}$ in (1), we find that

$$
r^{\alpha+a-2} \cdot B_{n} \cdot L_{n, k_{n}}=A_{n, k_{n}} \cdot u_{k_{n}} \cdots u_{n} .
$$

It then follows that $r^{\alpha+a-2} \mid A_{n, k_{n}}$, since the requirement $\left(r, u_{0}\right)=1$ implies that $\left(r, u_{k}\right)=1$ for all $0 \leq k \leq n$. Then, we obtain from (11) and Lemma 2.2 that

$$
L_{n, k_{n}} \geq r^{\alpha+a-2} C_{n, k_{n}} \geq u_{0} r^{\alpha}(r+1)^{n} .
$$

Theorem 1.2 follows.

As a corollary of Theorem 1.2, we obtain a substantial sharpening of Theorem 1.1 . 
Corollary 3.2. Fix integers $a \geq 2$ and $\beta \geq 1$. Then, for all but finitely many choices of integers $\alpha, r \geq a$, we have that $L_{n} \geq u_{0} r^{\alpha+\beta+a-2}(r+1)^{n}$ whenever $n>r^{\alpha}$.

Proof. By Theorem 1.2 we have $L_{n} \geq u_{0} r^{\alpha+\beta+a-2}(r+1)^{n}$ whenever $n \geq$ $2(\alpha+\beta+a-2) r$. If $r^{\alpha}+1 \geq 2(\alpha+\beta+a-2) r$, then the condition $n>r^{\alpha}$ guarantees that $n \geq 2(\alpha+\beta+a-2) r$. Since, for any given integer $\beta \geq 1$, we have $r^{\alpha}+1 \geq 2(\alpha+\beta+a-2) r$ for all but finitely many choices of $\alpha, r \geq a$, the result follows immediately.

The bound of Corollary 3.2 becomes effective even for small $\alpha$ and $r$. For example, the choices of $a=2$ and $\beta=1$ in Corollary 3.2 sharpen Theorem 1.1 by a factor of $r$ for all but six choices of $\alpha, r \geq 2$.

\section{Examples with $L_{n}<u_{0} r^{\alpha}(r+1)^{n}$}

In their article, Hong and Yang [HY08a asserted that their condition $n>r^{\alpha}$ is actually necessary for the bound $L_{n}>u_{0} r^{\alpha}(r+1)^{n}$ in Theorem 1.1. This assertion was accompanied by an example,

$$
u_{0}=r=2, \quad \alpha=3, \quad n=8,
$$

in which $L_{n}=5040<104976=u_{0} r^{\alpha}(r+1)^{n}$ (see Remark 3.1 of HY08a $)$. This example (6) not only satisfies $r^{\alpha}=8 \nless 8=n$, but also satisfies $2 \alpha r=12 \not \leq 8=n$. Unfortunately, (6) does not satisfy the condition $\left(u_{0}, r\right)=1$, so it does not actually suffice to demonstrate the necessity of the condition $n>r^{\alpha}$ in Theorem 1.1 when $r=2$ and $\alpha=3$.

As $2 \alpha r<r^{\alpha}+1$ for all but three choices of $\alpha, r \geq 2$, examples with $L_{n}<$ $u_{0} r^{\alpha}(r+1)^{n}$ and $n=r^{\alpha}$ are available for at most three choices of $\alpha, r \geq 2$. A computer search of all $u_{0}<n=r^{\alpha}$ with $\left(u_{0}, r\right)=1$ in these three cases 1 indicates that there exists only one example with $L_{n}<u_{0} r^{\alpha}(r+1)^{n},\left(u_{0}, r\right)=1$, and $r^{\alpha}=n$ :

$$
u_{0}=1, \quad r=\alpha=2, \quad n=4,
$$

in which $L_{4}=\operatorname{lcm}\{1,3,5,7,9\}=315<324=1 \cdot 2^{2}(2+1)^{4}$.

\section{ACKNowledgements}

The authors greatly appreciate the helpful comments and suggestions of Andrea J. Hawksley, Professor Noam D. Elkies, Daniel M. Kane, Shrenik N. Shah, and the editor, Professor Wen-Ching Winnie Li.

\section{AdDED AFTER POSTING}

The footnote on page 1 should read "The first author was partly supported by the National Science Foundation of China, Grant No. 10971145, and by the Program for New Century Excellent Talents in University, Grant No. NCET-06-0785."

Page 3 , line -4 should read:

$$
L_{n, k_{n}} \geq r^{\alpha+a-2} C_{n, k_{n}} \geq u_{0} r^{\alpha+a-2}(r+1)^{n} .
$$

\footnotetext{
${ }^{1}$ We need only consider the cases with $u_{0}<n$, as the proof of Lemma 3.1 shows that $\alpha r<n-k_{n}$ a priori - and so the result of Theorem 1.2 holds-whenever $u_{0} \geq n$.
} 


\section{REFERENCES}

[BKS02] P. Bateman, J. Kalb, and A. Stenger, Problem 10797: A limit involving least common multiples, Amer. Math. Monthly 109 (2002), 393-394.

[Far05] B. Farhi, Minorations non triviales du plus petit commun multiple de certaines suites finies d'entiers, C. R. Math. Acad. Sci. Paris, Ser. I 341 (2005), 469-474. MR2180812 (2006g:11006)

[Far07] , Nontrivial lower bounds for the least common multiple of some finite sequences of integers, J. Number Theory 125 (2007), 393-411. MR2332595 (2008i:11001)

[FK09] B. Farhi and D. Kane, New results on the least common multiple of consecutive integers, Proc. Amer. Math. Soc. 137 (2009), 1933-1939. MR2480273

[Han72] D. Hanson, On the product of the primes, Canad. Math. Bull. 15 (1972), 33-37. MR0313179 (47:1734)

[HF06] S. Hong and W. Feng, Lower bounds for the least common multiple of finite arithmetic progressions, C. R. Math. Acad. Sci. Paris, Ser. I 343 (2006), 695-698. MR2284695 (2007h:11004)

[HQ09] S. Hong and G. Qian, The least common multiple of consecutive terms in arithmetic progressions, arXiv:0903.0530, 2009.

[HY08a] S. Hong and Y. Yang, Improvements of lower bounds for the least common multiple of finite arithmetic progressions, Proc. Amer. Math. Soc. 136 (2008), 4111-4114. MR2431021

[HY08b] , On the periodicity of an arithmetical function, C. R. Math. Acad. Sci. Paris, Ser. I 346 (2008), 717-721. MR2427068 (2009e:11007)

[Nai82] M. Nair, On Chebyshev-type inequalities for primes, Amer. Math. Monthly 89 (1982), 126-129. MR643279 (83f:10043)

Mathematical College, Sichuan University, Chengdu 610064, People's Republic of CHINA

E-mail address: sfhong@scu.edu.cn, s-f.hong@tom.com, hongsf02@yahoo.com

Department of Mathematics and Department of Economics, Harvard University, Cambridge, Massachusetts 02138

Current address: Baker Library 420C, Harvard Business School, Soldiers Field, Boston, Massachusetts 02163

E-mail address: kominers@fas.harvard.edu, skominers@gmail.com

$U R L:$ http://www. scottkom.com/ 